\title{
Analysis via Passivity Theory of a Class of Nonlinear PID Global Regulators for Robot Manipulators
}

\author{
Jose Luis Mezaํㄹ, Víctor Santibáñez ${ }^{1}$, Rogelio Soto², \\ Jose Perez ${ }^{3}$ and Joel Perez ${ }^{3}$ \\ ${ }^{1}$ Instituto Tecnológico de la Laguna \\ ${ }^{2}$ Tecnológico de Monterrey, Campus Monterrey \\ 3Universidad Autónoma de Nuevo León \\ Mexico
}

\section{Introduction}

We present a simple global asymptotic stability analysis, by using passivity theory for a class of nonlinear PID regulators for robot manipulators. Nonlinear control structures based on the classical PID controller, which assure global asymptotic stability of the closed-loop system, have emerged. Some works that deal with global nonlinear PID regulators based on Lyapunov theory have been reported by (Arimoto, 1995a), (Kelly, 1998) and (Santibáñez \& Kelly, 1998). Recently, a particular case of the class of nonlinear PID global regulators originally proposed in (Santibáñez \& Kelly, 1998) was presented by (Sun et al., 2009). Few saturated PID controllers (that is, bounded PID controllers taking into account the actuator torque constraints) have been reported: for the case of semiglobal asymptotic stability, a saturated linear PID controller was presented in (Alvarez et al., 2003) and (Alvarez et al., 2008); for the case of global asymptotic stability, saturated nonlinear PID controllers were introduced in (Gorez, 1999), (Meza et al., 2005), (Santibáñez et al., 2008). The work introduced by (Gorez, 1999) was the first bounded PID-like controller in assuring global regulation; the latter works, introduced in (Meza et al., 2005) and (Santibáñez et al., 2008), also guarantee global regulation, but with the advantage of a controller structure which is simpler than that presented in (Gorez, 1999). A local adaptive bounded regulator was presented by (Laib, 2000). Recently a new saturated nonlinear PID regulator for robots has been proposed in (Santibáñez et al., 2010), the controller structure considers the saturation phenomena of the control computer, the velocity servo-drivers and the torque constraints of the actuators. The work in (Orrante et al., 2010) presents a variant of the work presented by (Santibáñez et al., 2010), where now the controller is composed by a saturated velocity proportional (P) inner loop, provided by the servo-driver, and a saturated position proportional-integral (PI) outer loop, supplied by the control computer.

In this chapter we use a passivity based approach to explain the results of global regulation of a class of nonlinear PID controllers proposed by (Santibáñez \& Kelly, 1998), that include the particular cases reported by (Arimoto, 1995a) and (Kelly, 1998). At the end of the 80's, it was established in (Kelly \& Ortega, 1988) and (Landau \& Horowitz, 1988) that the nonlinear 
dynamics of rigid robots describe a passivity mapping from torque input to velocity output. This property is know as the passive structure of rigid robots (Ortega \& Spong, 1989). The controller design methodology for robot manipulators introduced by (Takegaki \& Arimoto, 1981), also called energy shaping plus damping injection technique, allows to naturally split the controller tasks into potential energy shaping for stabilization at the desired equilibrium, and damping injection, to make this equilibrium attractive (Ortega et al., 1995b). As a feature of this kind of controllers, it can be shown that the passivity property, from a new input torque to output velocity, is preserved for robots in closed-loop with the energy shaping term and damping injection term of the controller. Furthermore, considering that corresponding feedback to the integral action define a passive mapping, then it is possible to use a passivity theorem of interconnected systems to explain the stability of a class of nonlinear PID global regulators for robots. The theorem used allows to conclude global asymptotic stability of the origin of an unforced feedback system, which is composed by the feedback interconnection of state strictly passive dynamic systems with a passive and zero state observable system.

The objective of this chapter is to present in a simple framework the global asymptotic stability analysis, by using passivity theory for a class of nonlinear PID regulators for robot manipulators. The remainder of this chapter is organized as follows: Section 2 summarizes the dynamics for rigid robots and also recalls some of their important properties. The rationale behind the energy shaping plus damping injection technique for rigid robots are given in Section 3. The class of nonlinear PID regulators is given in Section 4. In Section 5 we recall the definition of the passivity concepts for dynamical systems and we present the passivity theorem useful for asymptotic stability analysis of interconnected systems. In Section 6 we present a passivity analysis and application of passivity theorem to conclude global asymptotic stability. An Evaluation in simulation to verify the theoretical results is presented in Section 7. Finally, our conclusions are shown in Section 8. Throughout this chapter, the norm of a vector $x$ is defined as $\|x\|=\sqrt{x^{T} x}$ and that of a matrix $A$ is defined as the corresponding induced norm $\|A\|=\sqrt{\lambda_{\mathrm{M}}\left\{A^{T} A\right\}} . L_{2}^{n}$ and $L_{2 e}^{n}$ denote the space of $n$-dimensional square integrable functions and its extension, respectively.

\section{Robot dynamics}

For control design purposes, it is necessary to have a mathematical model that reveals the dynamical behavior of a system. Robots manipulators are articulated mechanical systems composed of links connected by joints. Links and joints are usually made as rigid as possible so as to achieve high precision in robot positioning. The joints are mainly of two types: revolute and prismatic. Its dynamic model is characterized by nonlinear coupled secondorder differential equations, which describe the temporal interactions of the joint motions in response to the inertial, centrifugal and Coriolis, gravitational and actuating torques or forces. The most commonly used equations to model the dynamics of a robot are the Euler-Lagrange and Newton-Euler formulations. Here we use the Euler - Lagrange formulation. In this section we consider robot manipulators formed by an open kinematic chain. We assume that all the links are joined together by revolute joints. In the absence of friction and other disturbances, the Lagrangian $\mathcal{L}(\boldsymbol{q}, \dot{\boldsymbol{q}})$ of a mechanical system is defined by

$$
\mathcal{L}(\boldsymbol{q}, \dot{\boldsymbol{q}})=\mathcal{K}(\boldsymbol{q}, \dot{\boldsymbol{q}})-\mathcal{U}(\boldsymbol{q})
$$


where $\mathcal{K}(\boldsymbol{q}, \dot{\boldsymbol{q}})$ y $\mathcal{U}(\boldsymbol{q})$ represent the kinetic and potential energy of the system respectively. The equation of motion of Euler - Lagrange for a manipulator of $n$ degrees of freedom are given by:

$$
\frac{d}{d t}\left(\frac{\partial \mathcal{L}(\boldsymbol{q}, \dot{\boldsymbol{q}})}{\partial \dot{\boldsymbol{q}}}\right)-\frac{\partial \mathcal{L}(\boldsymbol{q}, \dot{\boldsymbol{q}})}{\partial \boldsymbol{q}}=\boldsymbol{\tau}
$$

where $\tau \in \mathbb{R}^{n}$ is the vector of external generalized forces acting on each joint of robot, $q \in$ $\mathbb{R}^{n}$ represents the vector of generalized coordinates of the system, $\dot{\boldsymbol{q}}=\frac{d}{d t} \boldsymbol{q}$ is the vector of generalized velocities. In the case of a robot manipulator of $n$ degrees of freedom, kinetic energy is a quadratic function of the velocity vector $\dot{q}$ of the form:

$$
\mathcal{K}(\boldsymbol{q}, \dot{\boldsymbol{q}})=\frac{1}{2} \dot{\boldsymbol{q}}^{T} M(\boldsymbol{q}) \dot{\boldsymbol{q}}
$$

where $M(\boldsymbol{q}) \in \mathbb{R}^{n \times n}$ is the manipulator inertia matrix, which is symmetric and positive definite. The equations of motion of Euler - Lagrange provide the following dynamic model (Spong et al., 2006):

$$
M(\boldsymbol{q}) \ddot{\boldsymbol{q}}+\dot{M}(\boldsymbol{q}) \dot{\boldsymbol{q}}-\frac{1}{2} \frac{\partial}{\partial \boldsymbol{q}}\left(\dot{\boldsymbol{q}}^{T} M(\boldsymbol{q}) \dot{\boldsymbol{q}}\right)+\frac{\partial \mathcal{U}(\boldsymbol{q})}{\partial \boldsymbol{q}}=\boldsymbol{\tau}
$$

or in compact form, the dynamics of a serial $n$-link rigid robot can be written as:

$$
M(\boldsymbol{q}) \ddot{\boldsymbol{q}}+C(\boldsymbol{q}, \dot{\boldsymbol{q}}) \dot{\boldsymbol{q}}+\boldsymbol{g}(\boldsymbol{q})=\boldsymbol{\tau}
$$

where $C(\boldsymbol{q}, \dot{\boldsymbol{q}}) \dot{\boldsymbol{q}}$ and $\boldsymbol{g}(\boldsymbol{q})$ are given by:

$$
\begin{gathered}
C(\boldsymbol{q}, \dot{\boldsymbol{q}}) \dot{\boldsymbol{q}}=\dot{M}(\boldsymbol{q}) \dot{\boldsymbol{q}}-\frac{1}{2} \frac{\partial}{\partial \boldsymbol{q}}\left(\dot{\boldsymbol{q}}^{T} M(\boldsymbol{q}) \dot{\boldsymbol{q}}\right) \\
\boldsymbol{g}(\boldsymbol{q})=\frac{\partial \mathcal{U}(\boldsymbol{q})}{\partial \boldsymbol{q}} .
\end{gathered}
$$

$C(\boldsymbol{q}, \dot{\boldsymbol{q}}) \dot{\boldsymbol{q}} \in \mathbb{R}^{n}$ is the vector of centrifugal and Coriolis forces, and $g(\boldsymbol{q}) \in \mathbb{R}^{n}$ is the vector of gravitational forces or torques obtained as the gradient of the potential energy of $\operatorname{robot} \mathcal{U}(\boldsymbol{q})$. In terms of the state vector $\left[\begin{array}{ll}\boldsymbol{q}^{T} & \dot{\boldsymbol{q}}^{T}\end{array}\right]^{T}$, the robot dynamics given by equation (2) can be written as:

$$
\frac{d}{d t}\left[\begin{array}{l}
\boldsymbol{q} \\
\dot{\boldsymbol{q}}
\end{array}\right]=\left[\begin{array}{c}
\dot{\boldsymbol{q}} \\
M(\boldsymbol{q})^{-1}(\boldsymbol{q})[\boldsymbol{\tau}(t)-C(\boldsymbol{q}, \dot{\boldsymbol{q}}) \dot{\boldsymbol{q}}-\boldsymbol{g}(\boldsymbol{q})] .
\end{array}\right]
$$

\subsection{Planar robot of 2 degrees of freedom}

The equation of motion of Euler-Lagrange (1) for a robot of $n$ degrees of freedom can be equivalently written as:

$$
\frac{d}{d t}\left(\frac{\partial \mathcal{L}(\boldsymbol{q}, \dot{\boldsymbol{q}})}{\partial \dot{q}_{i}}\right)-\frac{\partial \mathcal{L}(\boldsymbol{q}, \dot{\boldsymbol{q}})}{\partial \dot{q}_{i}}=\tau_{i} \quad i=1, \cdots, n .
$$

In the particular case of a planar robot of $n=2$ degrees of freedom rotational joints, consider the diagram shown in Fig. 1 (Reyes \& Kelly, 2001). It is known that the compact form (2) of the dynamics of a robot for two degrees of freedom with rigid links can be written as: 


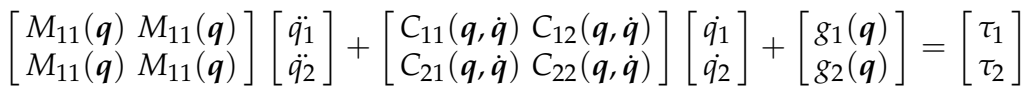

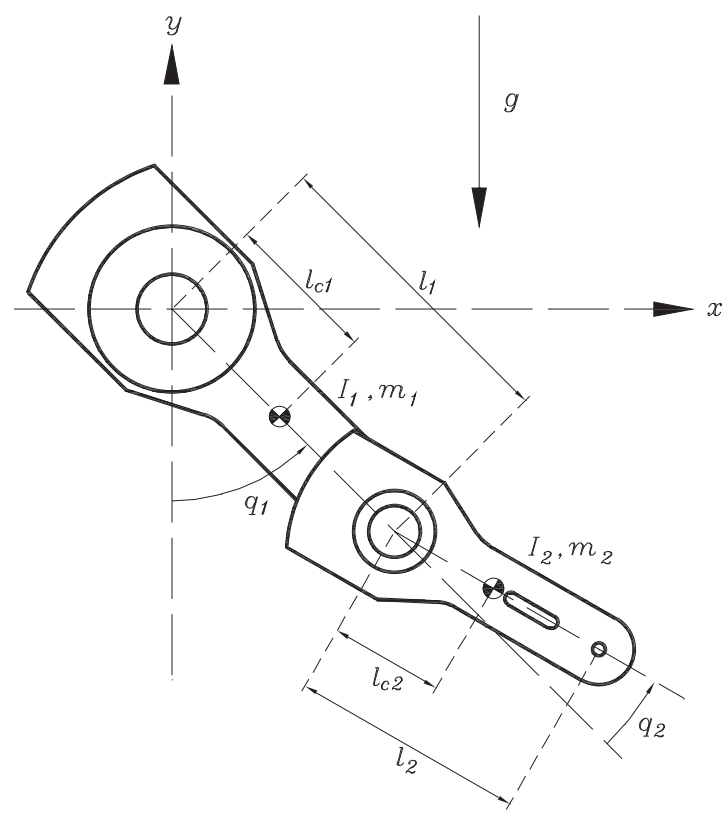

Fig. 1. Diagram of the prototype planar robot with 2 degrees of freedom.

The meaning of the parameters of the prototype planar robot is shown in Table 1.

\begin{tabular}{|c|c|}
\hline \hline Description & notation \\
\hline Angular displacement of link 1 & $q_{1}$ \\
\hline Angular displacement of link 2 & $q_{2}$ \\
\hline Length link 1 & $l_{1}$ \\
\hline Length link 2 & $l_{2}$ \\
\hline Link (1) center of mass & $l_{c 1}$ \\
\hline Link (2) center of mass & $l_{c 2}$ \\
\hline Mass link 1 & $m_{1}$ \\
\hline Mass link 2 & $m_{2}$ \\
\hline Inertia link 1 & $I_{1}$ \\
\hline Inertia link 2 & $I_{2}$ \\
\hline Gravity acceleration & $g$ \\
\hline
\end{tabular}

Table 1. Parameters of prototype planar robot with 2 degrees of freedom 
The elements of the inertia matrix $M(\boldsymbol{q})$ in terms of the parameters of the robot, are given by

$$
\begin{aligned}
& M_{11}(\boldsymbol{q})=m_{1} l_{c 1}^{2}+m_{2}\left(l_{1}^{2}+l_{c 2}^{2}+2 l_{1} l_{c 2} \cos \left(q_{2}\right)\right)+I_{1}+I_{2} \\
& M_{12}(\boldsymbol{q})=m_{2}\left(l_{c 2}^{2}+l_{1} l_{c 2} \cos \left(q_{2}\right)\right)+I_{2} \\
& M_{21}(\boldsymbol{q})=m_{2}\left(l_{c 2}^{2}+l_{1} l_{c 2} \cos \left(q_{2}\right)\right)+I_{2} \\
& M_{22}(\boldsymbol{q})=m_{2} l_{c 2}^{2}+I_{2} .
\end{aligned}
$$

The matrix elements $C_{i j}(\boldsymbol{q}, \dot{\boldsymbol{q}})(i, j=1,2)$ of centrifugal and Coriolis forces $C(\boldsymbol{q}, \dot{\boldsymbol{q}})$ are

$$
\begin{aligned}
& C_{11}(\boldsymbol{q}, \dot{\boldsymbol{q}})=-m_{2} l_{1} l_{c 2} \sin \left(q_{2}\right) \dot{q}_{2} \\
& C_{12}(\boldsymbol{q}, \dot{\boldsymbol{q}})=-m_{2} l_{1} l_{c 2} \sin \left(q_{2}\right)\left(\dot{q}_{1}+\dot{q}_{2}\right) \\
& C_{21}(\boldsymbol{q}, \dot{\boldsymbol{q}})=m_{2} l_{1} l_{c 2} \sin \left(q_{2}\right) \dot{q}_{1} \\
& C_{22}(\boldsymbol{q}, \dot{\boldsymbol{q}})=0 .
\end{aligned}
$$

Finally the elements of the vector of gravitational torques $g(\boldsymbol{q})$ are given by:

$$
\begin{aligned}
& g_{1}(\boldsymbol{q})=\left(m_{1} l_{c 1}+m_{2} l_{1}\right) g \sin \left(q_{1}\right)+m_{2} l_{c 2} g \sin \left(q_{1}+q_{2}\right) \\
& g_{2}(\boldsymbol{q})=m_{2} l_{c 2} g \sin \left(q_{1}+q_{2}\right) .
\end{aligned}
$$

\subsection{Properties of the robot dynamics}

Although the equation of motion (2) is complex, it has several fundamental properties which can be exploited to facilitate the analysis of stability. Three important properties of the robot dynamics are the following:

Property 1. (Koditschek, 1984) The matrix $C(\boldsymbol{q}, \dot{\boldsymbol{q}})$ and the time derivative $\dot{M}(\boldsymbol{q})$ of the inertia matrix satisfy:

$$
\dot{\boldsymbol{q}}^{T}\left[\frac{1}{2} \dot{M}(\boldsymbol{q})-C(\boldsymbol{q}, \dot{\boldsymbol{q}})\right] \dot{\boldsymbol{q}}=\mathbf{0} \quad \forall \boldsymbol{q}, \dot{\boldsymbol{q}} \in \mathbb{R}^{n} .
$$

Property 2. (Tomei, 1991) There exists a positive constant $k_{g}$ such that

$$
k_{g} \geq\left\|\frac{\partial \boldsymbol{g}(\boldsymbol{q})}{\partial \boldsymbol{q}}\right\| \forall \boldsymbol{q} \in \mathbb{R},
$$

and

$$
\|g(x)-g(y)\| \leq k_{g}\|x-y\| \quad \forall x, y \in \mathbb{R}^{n} .
$$

Property 3. (Passive structure of rigid robots) In relation to the dynamic model (2). The operator

$$
\begin{aligned}
H_{R} & : L_{2 e}^{n} \rightarrow L_{2 e}^{n} \\
& : \boldsymbol{\tau} \mapsto \dot{\boldsymbol{q}}
\end{aligned}
$$

is passive (Kelly \& Ortega, 1988; Landau \& Horowitz, 1988; Ortega \& Spong, 1989), i.e.:

$$
\underbrace{\int_{0}^{T} \dot{\boldsymbol{q}}(t)^{T} \boldsymbol{\tau}(t) d t}_{\text {applied-energy }}=\underbrace{V_{1}(T)-V_{1}(0)}_{\text {stored-energy }},
$$


which can be expressed as

$$
\int_{0}^{T} \dot{\boldsymbol{q}}(t)^{T} \boldsymbol{\tau}(t) d t \geq-V_{a}(0)
$$

where $V_{a}(t)$ is the total energy of robot plus a suitable constant which is introduced so that $V_{a}(t)$ is a nonnegative function (Ortega et al., 1995b):

$$
V_{a}(t)=\frac{1}{2} \dot{\boldsymbol{q}}(t)^{T} M(\boldsymbol{q}(t)) \dot{\boldsymbol{q}}(t)+\mathcal{U}(\boldsymbol{q}(t))-k_{u}
$$

being $\frac{1}{2} \dot{\boldsymbol{q}}^{T}(t) M(\boldsymbol{q}(t)) \dot{\boldsymbol{q}}(t)$ energy kinetic, $\mathcal{U}(\boldsymbol{q}(t))$ the potential energy of robot due to gravity, y

$$
k_{u}=\min _{\boldsymbol{q}} \mathcal{U}(\boldsymbol{q}(t)) .
$$

\section{Energy shaping methodology}

Energy is one of the fundamental concepts in control of mechanical systems with multidegrees-of-freedom. The action of a controller can be understood in energy terms as a dynamical system called "actuator" that supplies energies to the controlled system, upon interconnection, to modify desirably the behavior of the closed-loop (interconnected) system. This idea has its origin in (Takegaki \& Arimoto, 1981) and is later called the "energy-shaping" approach, which is now known as a basic controller design technique common in control of mechanical systems. Its systematic interpretation is called "passivity-based control" (Arimoto, 2009).

The main idea of this methodology is to reshape the robot system's natural energy and inject damping via velocity feedback, for asymptotic stabilization purposes, such that a regulation objective is reached. This is achieved by choosing a controller structure such that, first, the total potential energy function of the closed-loop system due to gravity and the controller is radially unbounded function in the position error with a unique and global minimum at zero position error, and second, it injects damping via velocity feedback. The resulting closed loop system is an autonomous one which has the nice property that zero position error and zero velocity form always the unique equilibrium point. By using the total energy, i.e., the kinetic plus total potential energy, as a Lyapunov function, it follows that this equilibrium is stable. In order to prove that the equilibrium is in fact globally asymptotically stable, the final key step is to exploit the autonomous nature of the closed loop system to invoke the Krasovskii-LaSalle's theorem. This approach has been continued by their colleagues and several researchers (Arimoto, 1995a; Nijmeijer \& Van der Schaft, 1990; Wen \& Bayard, 1988), who have offered extensions and improvements (Ailon \& Ortega, 1993; Berghuis \& Nijmeijer, 1993b; Kelly, 1993; Ortega et al., 1998) and (Ortega \& Garcia-Canseco, 2004; Ortega et al., 2008; Sepulchre et al., 1997; Vander, 1999).

As a feature of this kind of controllers, it can be shown that the passivity property, from a new input torque to output velocity, is preserved either for rigid robots or elastic joint robots in closed loop with the energy shaping term of the controller. The damping injection term, via velocity feedback or by way of a suitable filtering of position, defines an input, output or state strictly passive mapping from velocity input to damping output, and asymptotically stabilizes the desired equilibrium of the closed loop system.

We broach the regulation problem whose goal is to find $\tau(t)$ such that

$$
\lim _{t \rightarrow \infty} q(t)=q_{d}
$$


where $\boldsymbol{q}_{d} \in \mathbb{R}^{n}$ is a vector of constant desired joint displacements. The features of the system can be enhanced by reshaping its total potential energy. This can be done by constructing a controller to meet a desired energy function for the closed-loop system, and inject damping, via velocity feedback, for asymptotic stabilization purposes (Nijmeijer \& Van der Schaft, 1990). To this end, in this section we consider controllers whose control law can be written by

$$
\tau=\frac{\partial \mathcal{U}_{a}\left(\boldsymbol{q}_{d}, \tilde{\boldsymbol{q}}\right)}{\partial \tilde{\boldsymbol{q}}}-\frac{\partial \mathcal{F}(\dot{\boldsymbol{q}})}{\partial \dot{\boldsymbol{q}}}
$$

where $\mathcal{F}(\dot{\boldsymbol{q}})$ is some kind of dissipation function from which the damping force can be derived, an example is the so called Rayleigh dissipative function $\mathcal{F}(\dot{\boldsymbol{q}})=\frac{1}{2} \dot{\boldsymbol{q}}^{T} K_{v} \dot{\boldsymbol{q}}$, where $K_{v}$ is the matrix of coefficient of viscous friction, $\tilde{q}=q_{d}-q \in \mathbb{R}^{n}$ denotes the joint position error and $\mathcal{U}_{a}\left(\boldsymbol{q}_{d}, \tilde{\boldsymbol{q}}\right)$ is some kind of artificial potential energy provided by the controller whose properties will be established later. The first right hand side term of (9) corresponds to the energy shaping part and the other one to the damping injection part.

We assume the dissipation function $\mathcal{F}(\dot{\boldsymbol{q}})$ satisfies the following conditions:

$$
\begin{array}{r}
\frac{\partial \mathcal{F}(\dot{\boldsymbol{q}})}{\partial \dot{\boldsymbol{q}}}=\mathbf{0} \Leftrightarrow \dot{\boldsymbol{q}}=\mathbf{0} \\
\dot{\boldsymbol{q}}^{T} \frac{\partial \mathcal{F}(\dot{\boldsymbol{q}})}{\partial \dot{\boldsymbol{q}}}>0 \forall \dot{\boldsymbol{q}} \neq \mathbf{0} .
\end{array}
$$

The closed-loop system equation obtained by substituting the control law (9) into the robot dynamics (2) leads to

$$
\begin{aligned}
& \frac{d}{d t}\left[\begin{array}{l}
\tilde{\boldsymbol{q}} \\
\dot{\boldsymbol{q}}
\end{array}\right]= \\
& {\left[M^{-1}\left[\frac{\partial}{\partial \tilde{\boldsymbol{q}}}\left\{\mathcal{U}\left(\boldsymbol{q}_{d}-\tilde{\boldsymbol{q}}\right)+\mathcal{U}_{a}\left(\boldsymbol{q}_{d}, \tilde{\boldsymbol{q}}\right)\right\}-\frac{\partial \mathcal{F}(\dot{\boldsymbol{q}})}{\partial \dot{\boldsymbol{q}}}-C(\boldsymbol{q}, \dot{\boldsymbol{q}}) \dot{\boldsymbol{q}}\right]\right]}
\end{aligned}
$$

where (3) has been used. If the total potential energy $\mathcal{U}_{T}\left(\boldsymbol{q}_{d}, \tilde{\boldsymbol{q}}\right)$ of the closed-loop system, defined as the sum of the potential energy $\mathcal{U}(\boldsymbol{q})$ due to gravity plus the artificial potential energy $\mathcal{U}_{a}\left(\boldsymbol{q}_{d}, \tilde{\boldsymbol{q}}\right)$ introduced by the controller

$$
\mathcal{U}_{T}\left(\boldsymbol{q}_{d}, \tilde{\boldsymbol{q}}\right)=\mathcal{U}\left(\boldsymbol{q}_{d}-\tilde{\boldsymbol{q}}\right)+\mathcal{U}_{a}\left(\boldsymbol{q}_{d}, \tilde{\boldsymbol{q}}\right),
$$

is radially unbounded in $\tilde{\boldsymbol{q}}$, and $\tilde{\boldsymbol{q}}=\mathbf{0} \in \mathbb{R}^{n}$ is an unique minimum, which is global for all $\boldsymbol{q}_{d}$, then the origin $\left[\begin{array}{cc}\tilde{\boldsymbol{q}}^{T} & \dot{\boldsymbol{q}}^{T}\end{array}\right]^{T}=\mathbf{0} \in \mathbb{R}^{2 n}$ of the closed-loop system (13) is global and asymptotically stable (Takegaki \& Arimoto, 1981).

\section{A class of nonlinear PID global regulators}

\subsection{Classical PID regulators}

Conventional proportional-integral-derivative PID regulators have been extensively used in industry due to their design simplicity, inexpensive cost, and effectiveness. Most of the present industrial robots are controlled through PID regulators (Arimoto, 1995a). The classical version of the PID regulator can be described by the equation:

$$
\boldsymbol{\tau}=K_{p} \tilde{\boldsymbol{q}}-K_{v} \dot{\boldsymbol{q}}+K_{i} \int_{0}^{t} \tilde{\boldsymbol{q}}(\sigma) d \sigma
$$


where $K_{p}, K_{v}$ and $K_{i}$ are positive definite diagonal $n \times n$ matrices, and $\tilde{q}=q_{d}-\boldsymbol{q}$ denotes the position error vector. Even though the PID controller for robot manipulators has been very used in industrial robots (Arimoto, 1995a), there still exist open problems, that make interesting its study. A open problem is the lack of a proof of global asymptotic stability (Arimoto, 1994). The stability proofs shown until now are only valid in a local sense (Arimoto, 1994; Arimoto et al., 1990; Arimoto \& Miyazaki, 1983; Arimoto, 1996; Dorsey, 1991; Kelly, 1995; Kelly et al., 2005; Rocco, 1996; Wen, 1990) or, in the best of the cases, in a semiglobal sense (Alvarez et al., 2000; Meza et al., 2007). In (Ortega et al., 1995a), a so-called $\mathrm{PI}^{2} \mathrm{D}$ controller is introduced, which is based on a PID structure but uses a filter of the position in order to estimate the velocity of the joints, and adds a term which is the integral of such an estimate of the velocity (this added term motivates the name $\mathrm{PI}^{2} \mathrm{D}$ ); for this controller, semiglobal asymptotic stability was proved. To solve the global positioning problem, some globally asymptotically stable PID-like regulators have also been proposed (Arimoto, 1995a; Gorez, 1999; Kelly, 1998; Santibáñez \& Kelly, 1998), such controllers, however, are nonlinear versions of the classical linear PID. We propose a new global asymptotic stability analysis, by using passivity theory for a class of nonlinear PID regulators for robot manipulators. For the purpose of this chapter, it is convenient to recall the following definition presented in (Kelly, 1998).

Definition 1. $\mathcal{F}(m, \varepsilon, x)$ with $1 \geq m>0, \varepsilon>0$ and $x \in \mathbb{R}^{n}$ denotes the set of all continuous differentiable increasing functions $\operatorname{sat}(\boldsymbol{x})=\left[\begin{array}{llll}\operatorname{sat}\left(x_{1}\right) & \operatorname{sat}\left(x_{2}\right) & \cdots & \operatorname{sat}\left(x_{n}\right)\end{array}\right]^{T}$ such that

- $|x| \geq|\operatorname{sat}(x)| \geq m|x| \quad \forall x \in \mathbb{R}:|x|<\varepsilon$

- $\varepsilon \geq|\operatorname{sat}(x)| \geq m \varepsilon \quad \forall x \in \mathbb{R}:|x| \geq \varepsilon$

- $1 \geq \frac{d}{d x} \operatorname{sat}(x) \geq 0 \quad \forall x \in \mathbb{R}$

where $|\cdot|$ stands for the absolute value.

For instance, the nonlinear vector function $\operatorname{sat}(\tilde{\boldsymbol{q}})=\left[\begin{array}{llll}\operatorname{sat}\left(\tilde{q}_{1}\right) & \operatorname{sat}\left(\tilde{q}_{2}\right) & \cdots & \operatorname{sat}\left(\tilde{q}_{n}\right)\end{array}\right]^{\frac{T}{T}}$, considered in Arimoto (Arimoto, 1995a) whose entries are given by

$$
\operatorname{sat}(x)=\operatorname{Sin}(x)= \begin{cases}\sin (x) & \text { if }|x|<\pi / 2 \\ 1 & \text { if } x \geq \pi / 2 \\ -1 & \text { if } x \leq-\pi / 2\end{cases}
$$

belongs to set $\mathcal{F}(\sin (1), 1, x)$.

\subsection{A class of nonlinear PID controllers}

The class of nonlinear PID global regulators under study was proposed in (Santibáñez \& Kelly, 1998). The structure is based on the gradient of a $\mathcal{C}^{1}$ artificial potential function $\mathcal{U}_{a}(\tilde{\boldsymbol{q}})$ satisfying some typical features required by the energy shaping methodology (Takegaki \& Arimoto, 1981). The PID control law can be written by ( see Fig. 2).

$$
\boldsymbol{\tau}=\frac{\partial \mathcal{U}_{a}(\tilde{\boldsymbol{q}})}{\partial \tilde{\boldsymbol{q}}}-K_{v} \dot{\boldsymbol{q}}+K_{i} \int_{0}^{t}[\alpha \operatorname{sat}(\tilde{\boldsymbol{q}}(\sigma))+\dot{\tilde{\boldsymbol{q}}}(\sigma)] d \sigma
$$




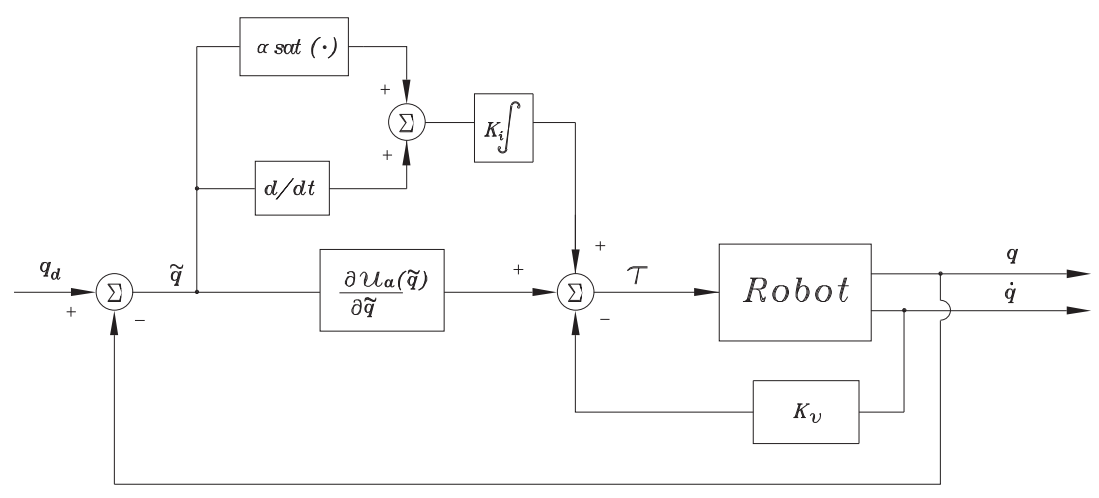

Fig. 2. Block diagram of nonlinear PID control

where

- $\mathcal{U}_{a}(\tilde{\boldsymbol{q}})$ is a kind of $\mathcal{C}^{1}$ artificial potential energy induced by a part of the controller.

- $K_{v}$ and $K_{i}$ are diagonal positive definite $n \times n$ matrices

- $\dot{\tilde{q}}$ is the velocity error vector

- $\operatorname{sat}(\tilde{\boldsymbol{q}}) \in \mathcal{F}(m, \varepsilon, \tilde{\boldsymbol{q}})$,

- $\alpha$ is a small constant, satisfying (Santibáñez \& Kelly, 1998)

By defining $z$ as:

$$
\boldsymbol{z}(t)=\int_{0}^{t}[\alpha \operatorname{sat}(\tilde{\boldsymbol{q}}(\sigma))+\dot{\tilde{\boldsymbol{q}}}(\sigma)] d \sigma-K_{p}^{-1} \boldsymbol{g}\left(\boldsymbol{q}_{d}\right),
$$

we can describe the closed-loop system by

$$
\begin{gathered}
\frac{d}{d t}\left[\begin{array}{c}
\tilde{\boldsymbol{q}} \\
\dot{\boldsymbol{q}} \\
z
\end{array}\right]= \\
{\left[\begin{array}{c}
-\dot{\boldsymbol{q}} \\
M(\boldsymbol{q})^{-1}\left[\begin{array}{c}
\left.\nabla_{\tilde{\boldsymbol{q}}} \mathcal{U}_{T}\left(\boldsymbol{q}_{d}, \tilde{\boldsymbol{q}}\right)-K_{v} \dot{\boldsymbol{q}}-C(\boldsymbol{q}, \dot{\boldsymbol{q}}) \dot{\boldsymbol{q}}+K_{i} z\right] \\
\alpha \operatorname{sat}(\tilde{\boldsymbol{q}})-\dot{\boldsymbol{q}}
\end{array}\right]
\end{array}\right.}
\end{gathered}
$$

which is an autonomous nonlinear differential equation whose origin $\left[\begin{array}{lll}\tilde{\boldsymbol{q}}^{T} & \dot{\boldsymbol{q}}^{T} & \boldsymbol{z}^{T}\end{array}\right]^{T}=\mathbf{0} \in \mathbb{R}^{3 n}$ is the unique equilibrium.

\subsection{Some examples}

Some examples of this kind of nonlinear PID regulators

$$
\boldsymbol{\tau}=\frac{\partial \mathcal{U}_{a}(\tilde{\boldsymbol{q}})}{\partial \tilde{\boldsymbol{q}}}-K_{v} \dot{\boldsymbol{q}}+K_{i} \int_{0}^{t}[\alpha \operatorname{sat}(\tilde{\boldsymbol{q}}(\sigma))+\dot{\tilde{\boldsymbol{q}}}(\sigma)] d \sigma
$$

are: 
- $\left(\right.$ Kelly, 1998) $\boldsymbol{\tau}=K_{p}^{\prime} \tilde{\boldsymbol{q}}-K_{v} \dot{\boldsymbol{q}}+K_{i}^{\prime} \int_{0}^{t} \operatorname{sat}(\tilde{\boldsymbol{q}}(\sigma)) d \sigma$ where $K_{p}^{\prime}=K_{p}+K_{p a}, K_{p a}$ is a diagonal positive definite $n \times n$ matrix with $\lambda_{\mathrm{m}}\left\{K_{p a}\right\}>k_{g}$, $K_{p}=K_{i}, K_{i}^{\prime}=\alpha K_{i}$. This controller has associated an artificial potential energy $\mathcal{U}_{a}(\tilde{\boldsymbol{q}})$ given by

$$
\mathcal{U}_{a}(\tilde{\boldsymbol{q}})=\frac{1}{2} \tilde{\boldsymbol{q}}^{T} K_{p a} \tilde{\boldsymbol{q}}
$$

- (Arimoto et al., 1994a) $\boldsymbol{\tau}=K_{p a} \operatorname{Sin}[\tilde{\boldsymbol{q}}]-K_{v} \dot{\boldsymbol{q}}+K_{i} \int_{0}^{t}[\alpha \operatorname{sat}(\tilde{\boldsymbol{q}}(\sigma))+\dot{\tilde{\boldsymbol{q}}}(\sigma)] d \sigma$ where $K_{p a}$ is a diagonal positive definite $n \times n$ matrix whose entries are $k_{\text {pai }}$ and $\operatorname{sat}(\tilde{\boldsymbol{q}})=$ $\operatorname{Sin}[\tilde{\boldsymbol{q}}]=\left[\operatorname{Sin}\left(\tilde{q}_{1}\right) \operatorname{Sin}\left(\tilde{q}_{2}\right) \ldots \operatorname{Sin}\left(\tilde{q}_{n}\right)\right]^{T}$ with $\operatorname{Sin}($.$) defined in (16).$

This controller has associated a $\mathcal{C}^{2}$ artificial potential energy $\mathcal{U}_{a}(\tilde{\boldsymbol{q}})$ given by

$$
\mathcal{U}_{a}(\tilde{\boldsymbol{q}})=\sum_{=1}^{n} k_{p a i}[1-\operatorname{Cos}(\tilde{\boldsymbol{q}})],
$$

where

$$
\operatorname{Cos}(x)= \begin{cases}\cos (x) & \text { if }|x|<\pi / 2 \\ -x+\pi / 2 & \text { if } x \geq \pi / 2 \\ x+\pi / 2 & \text { if } x \leq-\pi / 2\end{cases}
$$

- $\boldsymbol{\tau}=K_{p a} \tanh [\tilde{\boldsymbol{q}}]-K_{v} \dot{\boldsymbol{q}}+K_{i} \int_{0}^{t}[\alpha \operatorname{sat}(\tilde{\boldsymbol{q}}(\sigma))+\dot{\tilde{\boldsymbol{q}}}(\sigma)] d \sigma$

where $K_{p a}$ is a diagonal positive definite $n \times n$ matrix whose entries are $k_{p a i}$ and $\operatorname{sat}(\tilde{\boldsymbol{q}})=$ $\tanh [\tilde{\boldsymbol{q}}]=\left[\tanh \left(\tilde{q}_{1}\right) \tanh \left(\tilde{q}_{2}\right) \ldots \tanh \left(\tilde{q}_{n}\right)\right]^{T}$. This controller has associated a $\mathcal{C}^{\infty}$ artificial potential energy $\mathcal{U}_{a}(\tilde{\boldsymbol{q}})$ given by

$$
\mathcal{U}_{a}(\tilde{\boldsymbol{q}})=\sum_{i=1}^{n} k_{p a i} \ln \left[\cosh \left(\tilde{q}_{i}\right)\right]
$$

- $\boldsymbol{\tau}=K_{p a} \operatorname{Sat}[\tilde{\boldsymbol{q}}]-K_{v} \dot{\boldsymbol{q}}+K_{i} \int_{0}^{t}[\alpha \operatorname{sat}(\tilde{\boldsymbol{q}}(\sigma))+\dot{\tilde{\boldsymbol{q}}}(\sigma)] d \sigma$

where $K_{p a}$ is a diagonal positive definite $n \times n$ matrix whose entries are $k_{p a i}$ and $\operatorname{sat}(\tilde{\boldsymbol{q}})$ $=\operatorname{Sat}[\tilde{\boldsymbol{q}}]=\left[\operatorname{Sat}\left(\tilde{q}_{1}\right) \operatorname{Sat}\left(\tilde{q}_{2}\right) \ldots \operatorname{Sat}\left(\tilde{q}_{n}\right)\right]^{T}$. This controller has associated a $\mathcal{C}^{1}$ artificial potential energy $\mathcal{U}_{a}(\tilde{\boldsymbol{q}})$ given by

$$
\mathcal{U}_{a}\left(\boldsymbol{q}_{d}, \tilde{\boldsymbol{q}}\right)=\sum_{i=1}^{n}\left[\int_{0}^{\tilde{q}_{i}} k_{p a i} \operatorname{Sat}\left(\sigma_{i} ; \lambda_{i}\right) d \sigma_{i}\right]
$$

where Sat $(x ; \lambda)$ stands for the well known hard saturation function

$$
\operatorname{Sat}(x ; \lambda)=\left\{\begin{array}{l}
x \quad \text { if }\left|\tilde{q}_{i}\right|<\lambda \\
\lambda \quad \text { if } \tilde{q}_{i} \geq \lambda \\
-\lambda \text { if } \tilde{q}_{i} \leq-\lambda
\end{array} .\right.
$$

Following the ideas given in (Santibáñez \& Kelly, 1995) and (Loria et al., 1997) it is possible to demonstrate, for all above mentioned regulators, that $\mathcal{U}_{a}(\tilde{\boldsymbol{q}})$ leads to a radially unbounded virtual total potential function $\mathcal{U}_{T}\left(\boldsymbol{q}_{d}, \tilde{\boldsymbol{q}}\right)$. 


\section{Passivity concepts}

In this chapter, we consider dynamical systems represented by

$$
\begin{aligned}
& \dot{x}=f(x, u) \\
& y=h(x, u)
\end{aligned}
$$

where $\boldsymbol{u} \in \mathbb{R}^{n}, \boldsymbol{y} \in \mathbb{R}^{n}, x \in \mathbb{R}^{m}, f(\mathbf{0}, \mathbf{0})=\mathbf{0}$ and $\boldsymbol{h}(\mathbf{0}, \mathbf{0})=\mathbf{0}$. Moreover $f, \boldsymbol{h}$ are supposed sufficiently smooth such that the system is well-defined, i.e., $\forall \boldsymbol{u} \in L_{2 e}^{n}$ and $x(\mathbf{0}) \in \mathbb{R}^{m}$ we have that the solution $x(\cdot)$ is unique and $y \in L_{2 e}^{n}$.

Definition 2. (Khalil, 2002) The system (22)-(23) is said to be passive if there exists a continuously differentiable positive semidefinite function $V(x)$ (called the storage function) such that

$$
\boldsymbol{u}^{T} \boldsymbol{y} \geq \dot{V}(\boldsymbol{x})+\epsilon\|\boldsymbol{u}\|^{2}+\delta\|\boldsymbol{y}\|^{2}+\rho \psi(\boldsymbol{x})
$$

where $\epsilon, \delta$, and $\rho$ are nonnegative constants, and $\psi(x): \mathbb{R}^{m} \rightarrow \mathbb{R}$ is a positive definite function of $x$. The term $\rho \psi(x)$ is called the state dissipation rate. Furthermore, the system is said to be

- lossless if (24) is satisfied with equality and $\epsilon=\delta=\rho=0$; that is, $\boldsymbol{u}^{T} \boldsymbol{y}=\dot{V}(\boldsymbol{x})$

- input strictly passive if $\epsilon>0$ and $\delta=\rho=0$,

- output strictly passive if $\delta>0$ and $\epsilon=\rho=0$,

- state strictly passive if $\rho>0$ and $\epsilon=\delta=0$,

If more than one of the constants $\epsilon, \delta, \rho$ are positive we combine names.

Now we recall the definition of an observability property of the system (22)-(23).

Definition 3. (Khalil, 2002) The system (22)-(23) is said to be zero state observable if

$$
\boldsymbol{u}(t) \equiv \mathbf{0} \text { and } \boldsymbol{y}(t) \equiv \mathbf{0} \Rightarrow \boldsymbol{x}(t) \equiv \mathbf{0} .
$$

Equivalently, no solutions of $\dot{x}=f(\boldsymbol{x}, \mathbf{0})$ can stay identically in $S=\left\{\boldsymbol{x} \in \mathbb{R}^{m}: \boldsymbol{h}(\boldsymbol{x}, \mathbf{0})=\mathbf{0}\right\}$, other than the trivial solution $x(t) \equiv \mathbf{0}$.

Right a way, we present a theorem that allows to conclude global asymptotic stability for the origin of an unforced feedback system, which is composed by the feedback interconnection of a state strictly passive system with a passive system, which is an adaptation of a passivity theorem useful for asymptotic stability analysis of interconnected system presented in (Khalil, 2002).

Theorem 1. Consider the feedback system of Fig. 3 where $H_{1}$ and $H_{2}$ are dynamical systems of the form

$$
\begin{aligned}
\dot{x}_{i} & =f_{i}\left(\boldsymbol{x}_{i}, \boldsymbol{e}_{i}\right) \\
\boldsymbol{y}_{i} & =\boldsymbol{h}_{i}\left(\boldsymbol{x}_{i}, \boldsymbol{e}_{i}\right)
\end{aligned}
$$

for $i=1$, 2, where $f_{i}: \mathbb{R}^{m_{i}} \times \mathbb{R}^{n} \rightarrow \mathbb{R}^{m_{i}}$ and $\boldsymbol{h}_{i}: \mathbb{R}^{m_{i}} \times \mathbb{R}^{n} \rightarrow \mathbb{R}^{n}$ are supposed sufficiently smooth such that the system is well-defined. $f_{1}\left(\mathbf{0}, e_{1}\right)=0 \Rightarrow e_{1}=0, f_{2}(0,0)=0$, y $h_{i}(0,0)=0$. 


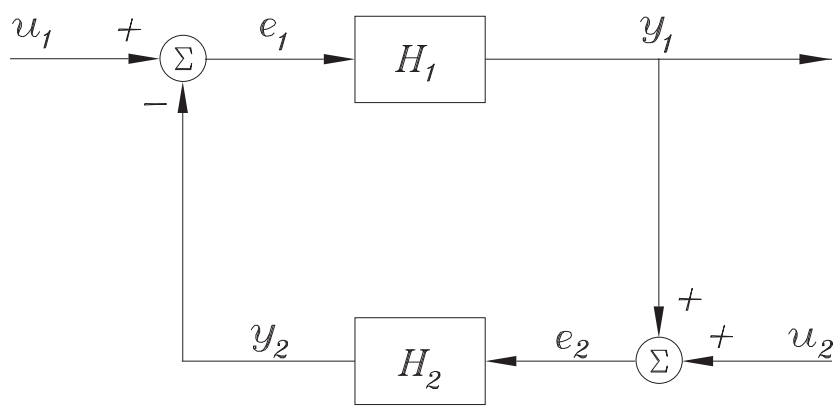

Fig. 3. Feedback connection

The system has the same number of inputs and outputs. Suppose the feedback system has a well-defined state-space model

$$
\begin{aligned}
& \dot{x}=f(x, u) \\
& y=h(x, u)
\end{aligned}
$$

where

$$
x=\left[\begin{array}{l}
x_{1} \\
x_{2}
\end{array}\right], u=\left[\begin{array}{l}
u_{1} \\
u_{2}
\end{array}\right], y=\left[\begin{array}{l}
y_{1} \\
y_{2}
\end{array}\right]
$$

$f$ and $\boldsymbol{h}$ are sufficiently smooth, $\boldsymbol{f}(\mathbf{0}, \mathbf{0})=\mathbf{0}$, and $\boldsymbol{h}(\mathbf{0}, \mathbf{0})=\mathbf{0}$. Let $H_{1}$ be a state strictly passive system with a positive definite storage function $V_{1}\left(x_{1}\right)$ and state dissipation rate $\rho_{1} \psi_{1}\left(x_{1}\right)$ and $\mathrm{H}_{2}$ be a passive and zero state observable system with a positive definite storage function $V_{2}\left(x_{2}\right)$; that is,

$$
\begin{aligned}
& \boldsymbol{e}_{1}^{T} \boldsymbol{y}_{1} \geq \dot{V}_{1}\left(x_{1}\right)+\rho_{1} \psi_{1}\left(x_{1}\right) \\
& \boldsymbol{e}_{2}^{T} \boldsymbol{y}_{2} \geq \dot{V}_{2}\left(x_{2}\right)
\end{aligned}
$$

Then the origin $x=0$ of

$$
\dot{x}=f(x, 0)
$$

is asymptotically stable. If $V_{1}\left(x_{1}\right)$ and $V_{2}\left(x_{2}\right)$ are radially unbounded then the origin of (25) will be globally asymptotically stable.

Proof. Take $\boldsymbol{u}_{1}=\boldsymbol{u}_{2}=\mathbf{0}$. In this case $\boldsymbol{e}_{1}=-\boldsymbol{y}_{2}$ and $\boldsymbol{e}_{2}=\boldsymbol{y}_{1}$. Using $V(\boldsymbol{x})=V_{1}\left(\boldsymbol{x}_{1}\right)+V_{2}\left(\boldsymbol{x}_{2}\right)$ as a Lyapunov function candidate for the closed-loop system, we have

$$
\begin{aligned}
\dot{V}(\boldsymbol{x}) & =\dot{V}_{1}\left(\boldsymbol{x}_{1}\right)+\dot{V}_{2}\left(\boldsymbol{x}_{2}\right) \\
& \leq \boldsymbol{e}_{1}^{T} \boldsymbol{y}_{1}-\rho_{1} \psi_{1}\left(\boldsymbol{x}_{1}\right)+\boldsymbol{e}_{2}^{T} \boldsymbol{y}_{2} \\
& =-\rho_{1} \psi_{1}\left(\boldsymbol{x}_{1}\right) \leq 0,
\end{aligned}
$$

which shows that the origin of the closed-loop system is stable. To prove asymptotic stability we use the LaSalle's invariance principle and the zero state observability of the system $H_{2}$. It remains to demonstrate that $\boldsymbol{x}=\mathbf{0}$ is the largest invariant set in $\Omega=\left\{\boldsymbol{x} \in \mathbb{R}^{m_{1}+m_{2}}: \dot{V}(\boldsymbol{x})=\right.$ 


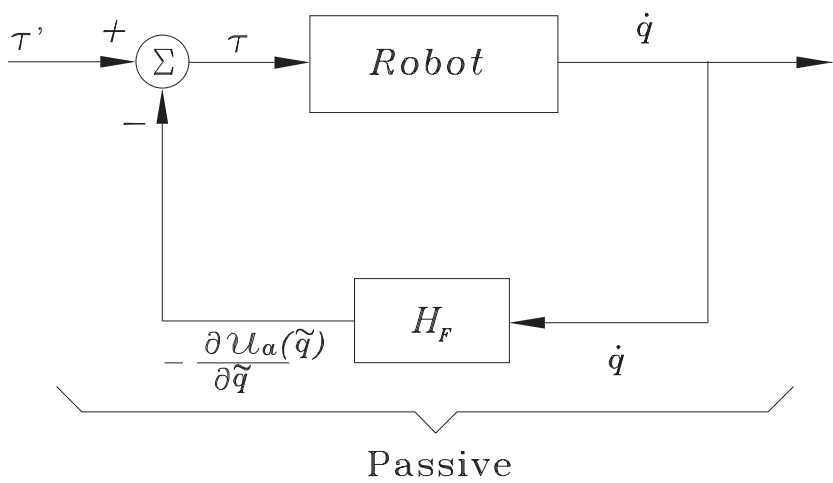

Fig. 4. Passivity structure of rigid robots in closed-loop

$0\}$. To this end, in the search of the largest invariant set, we have that $\dot{V}(\boldsymbol{x})=0 \Rightarrow 0 \leq$ $-\rho_{1} \psi_{1}\left(x_{1}\right) \leq 0 \Rightarrow-\rho_{1} \psi_{1}\left(x_{1}\right)=0$. Besides

$$
\rho_{1}>0 \Rightarrow \psi_{1}\left(x_{1}\right) \equiv 0 \Rightarrow x_{1} \equiv 0
$$

Now, as $x_{1} \equiv 0 \Rightarrow \dot{x}_{1}=f_{1} \equiv 0$ and in agreement with the assumption about $f_{1}$ in the sense that $f_{1}\left(0, e_{1}\right)=\mathbf{0} \Rightarrow e_{1}=\mathbf{0}$, we have $e_{1} \equiv \mathbf{0} \Rightarrow y_{2} \equiv \mathbf{0}$. Also $x_{1} \equiv \mathbf{0}, e_{1} \equiv \mathbf{0} \Rightarrow y_{1} \equiv \mathbf{0}$ (owing to assumption $\left.\boldsymbol{h}_{1}(\mathbf{0}, \mathbf{0})=\mathbf{0}\right)$. Finally, $y_{1} \equiv \mathbf{0} \Rightarrow \boldsymbol{e}_{2} \equiv \mathbf{0}$, and

$$
e_{2} \equiv \mathbf{0} \text { and } y_{2} \equiv \mathbf{0} \Rightarrow x_{2} \equiv \mathbf{0}
$$

in agreement with the zero state observability of $\mathrm{H}_{2}$. This shows that the largest invariant set in $\Omega$ is the origin, hence, by using the Krasovskii-LaSalle's theorem, we conclude asymptotic stability of the origin of the unforced closed-loop system (25). If $V(x)$ is radially unbounded then the origin will be globally asymptotically stable.

$\nabla \nabla \nabla$

\section{Analysis via passivity theory}

In this section we present our main result: the application of the passivity theorem given in Section 5, to prove global asymptotic stability of a class of nonlinear PID global regulators for rigid robots. First, we present two passivity properties of rigid robots in closed-loop with energy shaping based controllers.

Property 4. Passivity structure of rigid robots in closed-loop with energy shaping based controllers ( see Fig. 4). The system (2) in closed-loop with

$$
\tau=\frac{\partial \mathcal{U}_{a}\left(\boldsymbol{q}_{d}, \tilde{q}\right)}{\partial \tilde{q}}+\tau^{\prime}
$$

is passive, from input torque $\tau^{\prime}$ to output velocity $\dot{\boldsymbol{q}}$, with storage function

$$
\begin{aligned}
V(\dot{\boldsymbol{q}}, \tilde{\boldsymbol{q}})= & \frac{1}{2} \dot{\boldsymbol{q}}^{T} M(\boldsymbol{q}) \dot{\boldsymbol{q}}+\mathcal{U}_{T}\left(\boldsymbol{q}_{d}, \tilde{\boldsymbol{q}}\right) \\
& -\mathcal{U}_{T}\left(\boldsymbol{q}_{d}, \mathbf{0}\right),
\end{aligned}
$$


This is,

$$
\int_{0}^{T} \dot{\boldsymbol{q}}(t)^{T} \boldsymbol{\tau}^{\prime} d t \geq-V(\dot{\boldsymbol{q}}(0), \tilde{\boldsymbol{q}}(0)),
$$

where $\mathcal{U}_{a}\left(\boldsymbol{q}_{d}, \tilde{\boldsymbol{q}}\right)$ is the artificial potential energy introduced by the controller with properties requested by the energy shaping methodology and $\mathcal{U}_{T}\left(\boldsymbol{q}_{d}, \tilde{\boldsymbol{q}}\right)$ is the total potential energy of the closed-loop system, which has an unique minimum that is global.

Furthermore the closed-loop system is zero state observable.

Proof. The system (2) in closed-loop with control law (26) is given by

$$
\begin{gathered}
\frac{d}{d t}\left[\begin{array}{c}
\tilde{\boldsymbol{q}} \\
\dot{\boldsymbol{q}}
\end{array}\right]= \\
{\left[\begin{array}{c}
-\dot{\boldsymbol{q}} \\
M^{-1}(\boldsymbol{q})\left[\frac{\partial \mathcal{U}_{T}\left(\boldsymbol{q}_{d}-\tilde{\boldsymbol{q}}\right)}{\partial \tilde{\boldsymbol{q}}}-C(\boldsymbol{q}, \dot{\boldsymbol{q}}) \dot{\boldsymbol{q}}\right]+\left[M^{-1}(\boldsymbol{q}) \boldsymbol{\tau}^{\prime}\right]
\end{array}\right]}
\end{gathered}
$$

where (3) and (14) have been used. In virtue of Property 1, the time derivate of the storage function (27) along the trajectories of the closed-loop system (30) yields

$$
\dot{V}(\dot{\boldsymbol{q}}(t), \tilde{\boldsymbol{q}}(t))=\dot{\boldsymbol{q}}^{T} \boldsymbol{\tau}^{\prime}
$$

where integrating from 0 to $T$, in a direct form we obtain (28), thus, passivity from $\tau^{\prime}$ to $\dot{q}$ has been proved.

The zero state observability property of the system (30) can be proven, by taking the output as $\boldsymbol{y}=\dot{\boldsymbol{q}}$ and the input as $\boldsymbol{u}=\boldsymbol{\tau}^{\prime}$, because

$$
\dot{\boldsymbol{q}} \equiv \mathbf{0}, \boldsymbol{\tau}^{\prime} \equiv \mathbf{0} \Rightarrow \tilde{\boldsymbol{q}} \equiv \mathbf{0} .
$$

The robot passive structure is preserved in closed-loop with the energy shaping based controllers, because this kind of controllers also have a passive structure. Passivity is invariant for passive systems which are interconnected in closed-loop, and the resulting system is also passive.

Property 5. State strictly passivity of rigid robots in closed-loop with the energy shaping plus damping injection based regulators (see Fig. 5). The system (2) in closed-loop with

$$
\boldsymbol{\tau}=\frac{\partial \mathcal{U}_{a}\left(\boldsymbol{q}_{d}, \tilde{\boldsymbol{q}}\right)}{\partial \tilde{\boldsymbol{q}}}-K_{v} \dot{\boldsymbol{q}}+\boldsymbol{\tau}^{\prime \prime}
$$

is state strictly passive, from input torque $\boldsymbol{\tau}^{\prime \prime}$ to output $(\dot{\boldsymbol{q}}-\alpha \operatorname{sat}(\tilde{\boldsymbol{q}}))$, with storage function

$$
\begin{aligned}
V(\dot{\boldsymbol{q}}, \tilde{\boldsymbol{q}})= & \frac{1}{2} \dot{\boldsymbol{q}}^{T} M(\boldsymbol{q}) \dot{\boldsymbol{q}}+\mathcal{U}_{T}\left(\boldsymbol{q}_{d}, \tilde{\boldsymbol{q}}\right) \\
& -\mathcal{U}_{T}\left(\boldsymbol{q}_{d}, \mathbf{0}\right)-\alpha \operatorname{sat}(\tilde{\boldsymbol{q}})^{T} M(\boldsymbol{q}) \dot{\boldsymbol{q}},
\end{aligned}
$$

where $\frac{1}{2} \dot{\boldsymbol{q}}^{T} M(\boldsymbol{q}) \dot{\boldsymbol{q}}$ is the kinetic energy, $\mathcal{U}_{T}\left(\boldsymbol{q}_{d}, \tilde{\boldsymbol{q}}\right)$ is the total potential energy of the closedloop system, and $\alpha \operatorname{sat}(\tilde{\boldsymbol{q}}) M(\boldsymbol{q}) \dot{\boldsymbol{q}}$ is a cross term which depends on position error and velocity, 


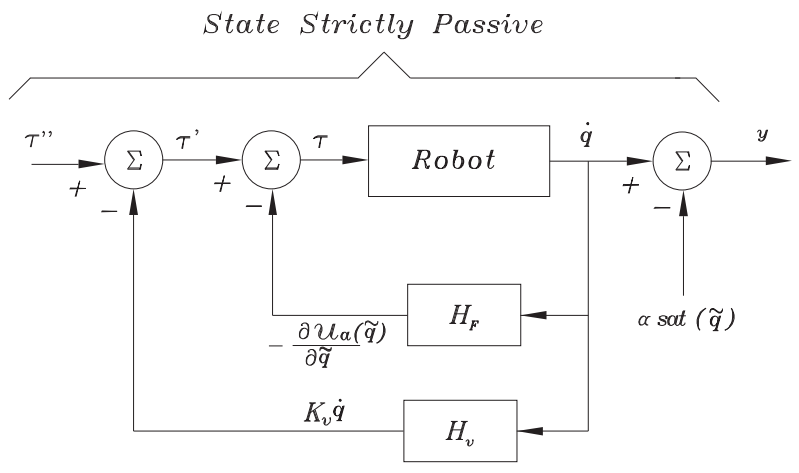

Fig. 5. State strictly passivity of rigid robots in closed-loop with energy shaping plus damping injection based regulator

and $\alpha$ is a small constant (Santibáñez \& Kelly, 1998). In this case $K_{v} \dot{\boldsymbol{q}}$ is the damping injection term. The State dissipation rate is given by :

$$
\begin{aligned}
\varphi(\dot{\boldsymbol{q}}, \tilde{\boldsymbol{q}})= & \dot{\boldsymbol{q}}^{T} K_{v} \dot{\boldsymbol{q}}+\alpha \operatorname{sat}(\tilde{\boldsymbol{q}}) M(\boldsymbol{q}) \dot{\boldsymbol{q}} \\
& -\alpha \operatorname{sat}(\tilde{\boldsymbol{q}}) C(\tilde{\boldsymbol{q}}, \dot{\boldsymbol{q}})^{T} \dot{\boldsymbol{q}} \\
& -\alpha \operatorname{sat}(\tilde{\boldsymbol{q}}) K_{p} \tilde{\boldsymbol{q}}+\alpha \operatorname{sat}(\tilde{\boldsymbol{q}}) K_{v} \dot{\boldsymbol{q}}
\end{aligned}
$$

Consequently the inner product of the input $\boldsymbol{\tau}^{\prime \prime}$ and the output $y=(\dot{\boldsymbol{q}}-\alpha \operatorname{sat}(\tilde{\boldsymbol{q}}))$ is given by:

$$
(\dot{\boldsymbol{q}}-\alpha \operatorname{sat}(\tilde{\boldsymbol{q}}))^{T} \boldsymbol{\tau}^{\prime \prime} \geq \dot{V}(\dot{\boldsymbol{q}}, \tilde{\boldsymbol{q}})+\varphi(\dot{\boldsymbol{q}}, \tilde{\boldsymbol{q}}),
$$

Proof. The closed-loop system(2) with control law (31) is

$$
\begin{gathered}
\frac{d}{d t}\left[\begin{array}{c}
\tilde{\boldsymbol{q}} \\
\dot{\boldsymbol{q}}
\end{array}\right]= \\
{\left[\begin{array}{c}
-\dot{\boldsymbol{q}} \\
M^{-1}(\boldsymbol{q})\left[\frac{\partial \mathcal{U}_{T}\left(\boldsymbol{q}_{d}-\tilde{\boldsymbol{q}}\right)}{\partial \tilde{\boldsymbol{q}}}-K_{v} \dot{\boldsymbol{q}}-C(\boldsymbol{q}, \dot{\boldsymbol{q}}) \dot{\boldsymbol{q}}\right]+\left[M^{-1}(\boldsymbol{q}) \boldsymbol{\tau}^{\prime \prime}\right]
\end{array}\right]}
\end{gathered}
$$

where (3) and (14) have been used. In virtue of property 1, the time derivate of the storage function (32) along the trajectories of the closed-loop system (36) yields to

$$
\dot{V}(\dot{\boldsymbol{q}}(t), \tilde{\boldsymbol{q}}(t))=(\dot{\boldsymbol{q}}-\alpha \operatorname{sat}(\tilde{\boldsymbol{q}}))^{T} \boldsymbol{\tau}^{\prime \prime}-\varphi(\dot{\boldsymbol{q}}(t), \tilde{\boldsymbol{q}}(t)),
$$

from which we get (34), so state strictly passivity from input $\tau^{\prime \prime}$ to output $(\dot{\boldsymbol{q}}-\alpha \operatorname{sat}(\tilde{\boldsymbol{q}}))$ is proven.

The robot dynamics enclosed loop with the energy shaping plus damping injection based controllers defines a state strictly passive mapping, from torque input $\boldsymbol{\tau}^{\prime \prime}$ to output $y=(\dot{\boldsymbol{q}}-$ $\alpha \operatorname{sat}(\tilde{\boldsymbol{q}}))$

$$
y^{T} \boldsymbol{\tau}^{\prime \prime} \geq \dot{V}_{1}(\dot{\boldsymbol{q}}(t), \tilde{\boldsymbol{q}}(t))+\varphi(\dot{\boldsymbol{q}}(t), \tilde{\boldsymbol{q}}(t)),
$$

where $\varphi(\dot{\boldsymbol{q}}, \tilde{\boldsymbol{q}})$ is called the state dissipation rate given by (33) with a storage function 


$$
\begin{array}{r}
V_{1}(\tilde{\boldsymbol{q}}, \dot{\boldsymbol{q}})=\frac{1}{2} \dot{\boldsymbol{q}}^{T} M(\boldsymbol{q}) \dot{\boldsymbol{q}}+\mathcal{U}_{T}\left(\boldsymbol{q}_{d}, \tilde{\boldsymbol{q}}\right)-\mathcal{U}_{T}\left(\boldsymbol{q}_{d}, \mathbf{0}\right) \\
-\alpha \operatorname{sat}(\tilde{\boldsymbol{q}})^{T} M(\boldsymbol{q}) \dot{\boldsymbol{q}},
\end{array}
$$

which is positive definite function and radially unbounded (Santibáñez \& Kelly, 1995).

The integral action defines a zero state observable passive mapping with a radially unbounded and positive definite storage function

$$
V_{2}(z)=\frac{1}{2} z^{T} K_{i} z
$$

By considering the robot dynamics in closed loop with the energy shaping plus damping injection based control action, in the forward path and the integral action in the feedback path (see Fig. 6), then, the feedback system satisfies in a direct way the theorem 1 conditions and we conclude global asymptotic stability of the closed loop system.

State Strictly Possive

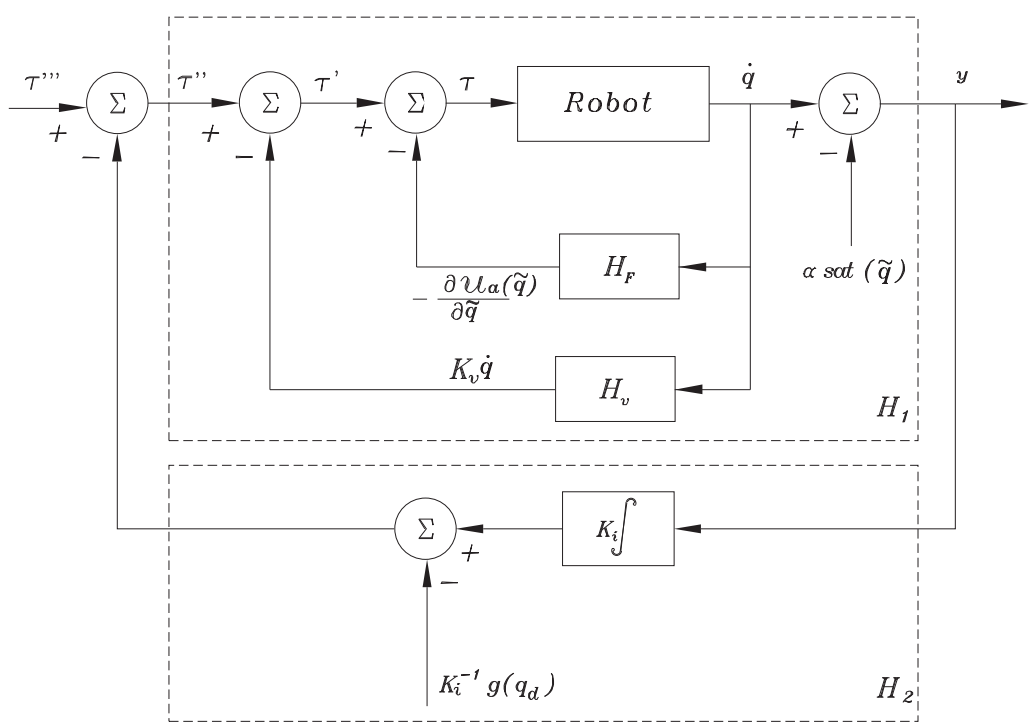

Passive and Zero State Observable

Fig. 6. Robot dynamics with Nonlinear PID controller

So we have proved the following:

\section{Proposition 1.}

Consider the class of nonlinear PID regulators (17) in closed-loop with robot dynamics (2). The closed-loop system can be represented by an interconnected system, which satisfies the following conditions 
- A1. The system in the forward path defines a state strictly passive mapping with a radially unbounded positive definite storage function.

- A2. The system in the feedback path defines a zero state observable passive mapping with a radially unbounded positive definite storage function.

Besides, the equilibrium $\left[\begin{array}{lll}\tilde{\boldsymbol{q}}^{T} & \dot{\boldsymbol{q}}^{T} & \boldsymbol{z}^{T}\end{array}\right]^{T}=\mathbf{0} \in \mathbb{R}^{3 n}$ of the closed-loop system (20) is globally asymptotically stable.

\section{Simulation results}

Computer simulations have been carried out to illustrate the performance of a class of nonlinear PID global regulators for robot manipulators. A example of this kind of nonlinear PID regulators is given by (Kelly, 1998)

$$
\boldsymbol{\tau}=K_{p} \tilde{\boldsymbol{q}}-K_{v} \dot{\boldsymbol{q}}+K_{i} \int_{0}^{t}[\alpha \operatorname{sat}(\tilde{\boldsymbol{q}}(\sigma))+\dot{\tilde{\boldsymbol{q}}}(\sigma)] d \sigma
$$

where artificial potential energy is given by $\mathcal{U}_{a}(\tilde{\boldsymbol{q}})=\frac{1}{2} \tilde{\boldsymbol{q}}^{T} K_{p} \tilde{\boldsymbol{q}}$, hence $\frac{\partial \mathcal{U}_{a}(\tilde{\boldsymbol{q}})}{\partial \tilde{\boldsymbol{q}}}=K_{p} \tilde{\boldsymbol{q}}$.

The manipulator used for simulation is a two revolute joined robot (planar elbow manipulator), as show in Fig. 1. The meaning of the symbols is listed in Table 2 whose numerical values have been taken from (Reyes \& Kelly, 2001).

\begin{tabular}{|c|c|c|c|}
\hline \hline Parameters & Notation & Value & Unit \\
\hline Length link 1 & $l_{1}$ & 0.45 & $\mathrm{~m}$ \\
\hline Length link 2 & $l_{2}$ & 0.45 & $\mathrm{~m}$ \\
\hline Link (1) center of mass & $l_{c 1}$ & 0.091 & $\mathrm{~m}$ \\
\hline Link (2) center of mass & $l_{c 2}$ & 0.048 & $\mathrm{~m}$ \\
\hline Mass link 1 & $m_{1}$ & 23.902 & $\mathrm{~kg}$ \\
\hline Mass link 2 & $m_{2}$ & 3.88 & $\mathrm{~kg}$ \\
\hline Inertia link 1 & $I_{1}$ & 1.266 & $\mathrm{Kg} \mathrm{m}^{2} / \mathrm{rad}$ \\
\hline Inertia link 2 & $I_{2}$ & 0.093 & $\mathrm{Kg} \mathrm{m}^{2} / \mathrm{rad}$ \\
\hline Gravity acceleration & $g$ & 9.81 & $\mathrm{~m} / \mathrm{s}^{2}$ \\
\hline
\end{tabular}

Table 2. Physical parameters of the prototype planar robot with 2 degrees of freedom

The entries of the dynamics of this two degrees-of-freedom direct-drive robotic arm are given by (Meza et al., 2007):

$$
\begin{gathered}
M(\boldsymbol{q})=\left[\begin{array}{cc}
2.351+0.168 \cos \left(q_{2}\right) & 0.102+0.084 \cos \left(q_{2}\right) \\
0.102+0.084 \cos \left(q_{2}\right) & 0.102
\end{array}\right] \\
C(\boldsymbol{q}, \dot{\boldsymbol{q}})=\left[\begin{array}{cc}
-0.084 \sin \left(q_{2}\right) \dot{q}_{2} & -0.084 \sin \left(q_{2}\right)\left(\dot{q}_{1}+\dot{q}_{2}\right) \\
0.084 \sin \left(q_{2}\right) \dot{q}_{1} & 0
\end{array}\right] \\
g(\boldsymbol{q})=9.81\left[\begin{array}{c}
3.921 \sin \left(q_{1}\right)+0.186 \sin \left(q_{1}+q_{2}\right) \\
0.186 \sin \left(q_{1}+q_{2}\right)
\end{array}\right]
\end{gathered}
$$


The PID tuning method is based on the stability analysis presented in (Santibáñez \& Kelly, 1998). The tuning procedure for the PID controller gains can be written as:

$$
\begin{array}{r}
\lambda_{\mathrm{M}}\left\{K_{i}\right\} \geq \lambda_{\mathrm{m}}\left\{K_{i}\right\}>0 \\
\lambda_{\mathrm{M}}\left\{K_{v}\right\} \geq \lambda_{\mathrm{m}}\left\{K_{v}\right\}>0 \\
\lambda_{\mathrm{M}}\left\{K_{p}\right\} \geq \lambda_{\mathrm{m}}\left\{K_{p}\right\}>k_{g}
\end{array}
$$

where $K_{p}$ denotes a diagonal positive definite $n \times n$ gain matrix resulting of the artificial potential energy $\mathcal{U}_{a}(\tilde{\boldsymbol{q}})=\frac{1}{2} \tilde{\boldsymbol{q}}^{T} K_{p} \tilde{\boldsymbol{q}}$ of the controller. The PID tuning requires to compute $k_{g}$. Using property 2 and the above expressions of the gravitational torque vector, we obtain that $k_{g}=80.578 \quad\left[\mathrm{~kg} \mathrm{~m}{ }^{2} / \mathrm{sec}^{2}\right]$.

The gain was tuned as $K_{p}=\operatorname{diag}\{130,81\}[\mathrm{Nm} / \mathrm{rad}], K_{i}=\operatorname{diag}\{30,5\}[\mathrm{Nm} / \mathrm{rad}$ sec $]$ and $K_{v}=\operatorname{diag}\{31,18\}[\mathrm{Nm} \mathrm{sec} / \mathrm{rad}]$ and $\alpha=1$. The maximum torques supplied by the actuators are $\tau_{1}^{\max }=150[\mathrm{Nm}]$ and $\tau_{2}^{\max }=15[\mathrm{Nm}]$. With the end of supporting the effectiveness of the proposed controller we have used a squared signal whose amplitude is decreased in magnitude every two seconds. More specifically, the robot task is coded in the following desired joint positions

$$
\begin{array}{r}
\mathbf{q}_{\mathbf{d}_{\mathbf{1}}}(t)=\left\{\begin{aligned}
45 & \text { degrees if } 0 \leq t<2 \mathrm{sec} \\
30 & \text { degrees if } 2 \leq t<4 \mathrm{sec} \\
20 & \text { degrees if } 4 \leq t<6 \mathrm{sec} \\
0 & \text { degrees if } 6 \leq t<8 \mathrm{sec}
\end{aligned}\right. \\
\mathbf{q}_{\mathbf{d}_{\mathbf{2}}}(t)=\left\{\begin{aligned}
15 & \text { degrees if } 0 \leq t<2 \mathrm{sec} \\
10 & \text { degrees if } 2 \leq t<4 \mathrm{sec} \\
5 & \text { degrees if } 4 \leq t<6 \mathrm{sec} \\
0 & \text { degrees if } 6 \leq t<8 \mathrm{sec}
\end{aligned}\right.
\end{array}
$$

Above position references are piecewise constant and really demand large torques to reach the amplitude of the respective requested step. In order to evaluate the effectiveness of the proposed controller. The proposed Nonlinear PID control scheme has been tuned to get their best performance in the presence of a step input whose amplitude is $45 \mathrm{deg}$ for link 1 and 15 deg for link 2. The simulations results are depicted in Figs. (7)-(10), they show the desired and actual joint positions and the applied torques for the nonlinear PID control. From Figs. (7)-(8), one can observe that the transient for the nonlinear PID in each change of the step magnitude, of the links are really good and the accuracy of positioning is satisfactory.

Applied torque $\tau_{1}$ and $\tau_{2}$ are sketched in Figs. (9)-(10) these figures show the evolution of the applied torques to the robot joints during the execution of the simulations. Notice that initial torque peaks fit to the nominal torque limits.

\section{Conclusions}

In this chapter we have given sufficient conditions for global asymptotic stability of a class of nonlinear PID type controllers for rigid robot manipulators. By using a passivity approach, we have presented the asymptotic stability analysis based on the energy shaping methodology. The analysis has been done by using an adaptation of a passivity theorem presented in the literature. This passivity theorem, deals with systems composed by the feedback interconnection of a state strictly passive system with a passive system. Simulation results confirm that the class of nonlinear PID type controllers for rigid robot manipulators 


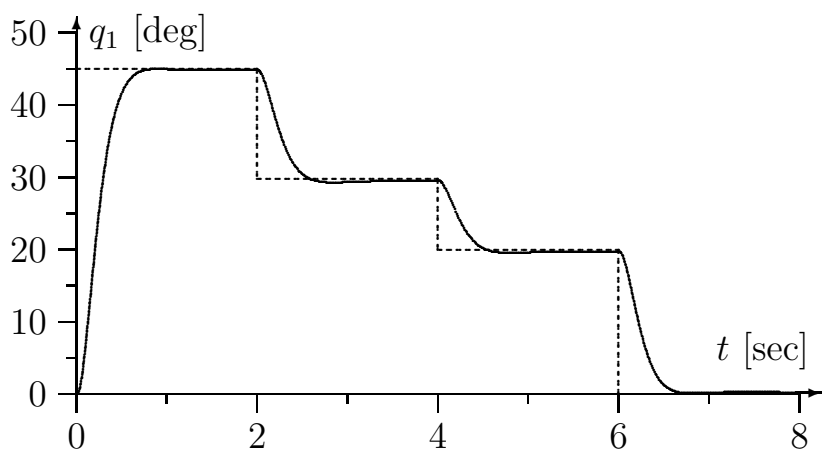

Fig. 7. Desired and actual positions 1 for the Nonlinear PID control

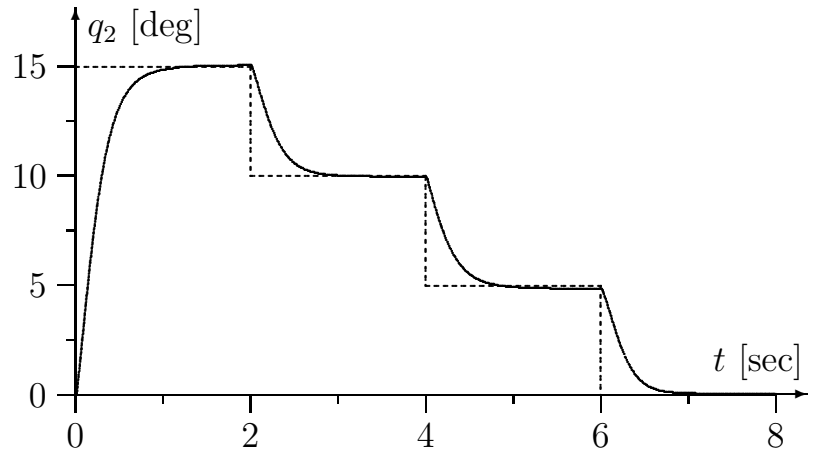

Fig. 8. Desired and actual positions 2 for the Nonlinear PID control

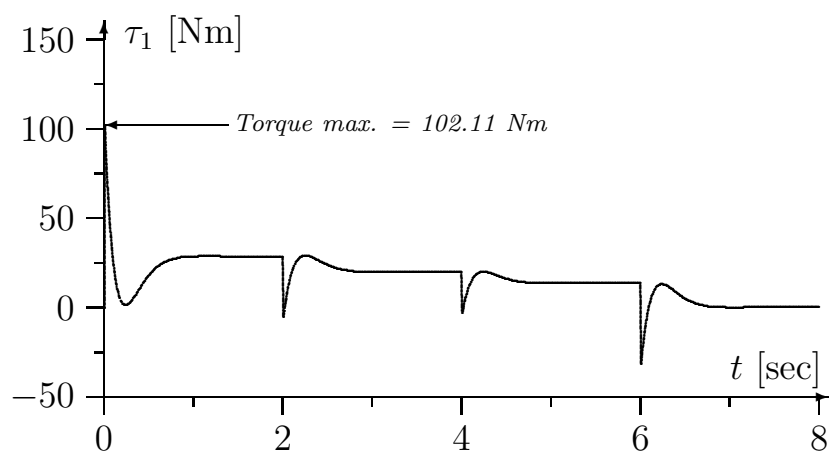

Fig. 9. Applied torque $\tau_{1}$ Nonlinear PID

have a good precision. The performance of the nonlinear PID type controllers has been 


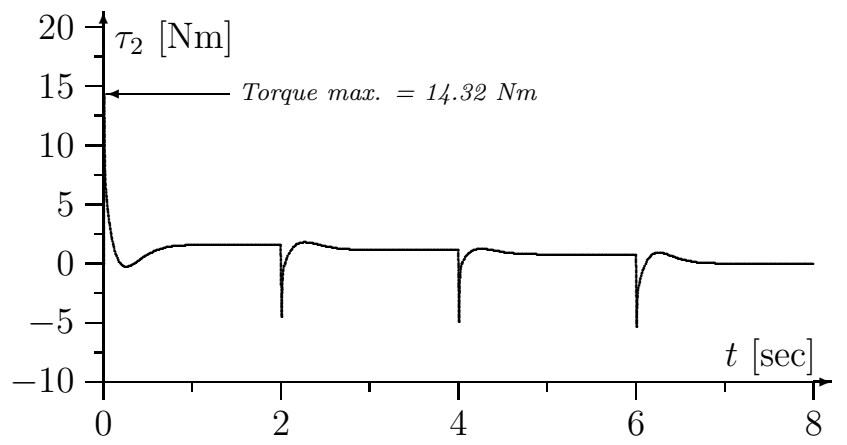

Fig. 10. Applied torque $\tau_{2}$ Nonlinear PID

verified on a two degree of freedom direct drive robot arm.

\section{Acknowledgment}

The authors would like to thank CONACYT(México) Grant No. 134534, Promep, Cátedra e-robots Tecnológico de Monterrey, DGEST for their support.

\section{References}

Ailon, A. \& Ortega, R. (1993) "An observer-based set-point controller for robot manipulators with flexible joints", Systems and Control Letters, North Holland, 21, pp. 329-335.

Alvarez, J.; Cervantes, I. \& Kelly, R. (2000). PID regulation of robot manipulators: stability and performance Systems and Control Letters, 41, 73-83.

Alvarez, J.; Kelly, R. \& Cervantes, I. (2003). Semiglobal stability of saturated linear PID control for robot manipulators. Automatica, Vol. 39, pp. 989-995.

Alvarez, J.; Santibáñez, V. \& Campa, R. (2008). Stability of robot manipulators under saturated PID compensation. IEEE Transactions on Control Systems Technology, Vol. 16, No. 6, pp. 1333-1341.

Arimoto, S.; Naniwa, T.; Parra-Vega, V. \& Whitcomb, L. (1994), A quasi-natural potential and its role in design of hyper-stable PID servo-loop for robotic systems, In Proc. of the CAI Pacific Symposium' 94 on Control and Industrial Automation Application, Hong Kong, pp. 110-117.

Arimoto, S. (1994). State of the art and future research directions of robot control, 4Th IFAC Symposium on Robot Control, Italy.

Arimoto, S.; Naniwa, T. \& Suzuki H.(1990), Asymptotic stability and robustness of PID local feedback for position control of robot manipulators, proceeding of ICARCV 90, Singapore 1990, 19-27.

Arimoto, S. \& Miyazaki, F. (1983). Stability and robustness of PID feedback control for robot manipulators of sensory capability, Robotics Researchs, First International Symposium MIT press, Cambridge, MA.

Arimoto, S. (1995a) "Fundamental problems of robot control: Part I, Innovations in the realm of robot servo-loops", Robotica, Vol. 13, pp. 19-27. 
Arimoto, S. (1996) Control theory of non-linear mechanical systems: A passivity-based and circuittheoretic approach, Oxford University Press.

Arimoto, S. (2009) Control of mechanical systems. Scholarpedia, 4(4):6520.

Berghuis, H. \& Nijmeijer, H. (1993b) "Global regulation of robots using only position measurement", Systems and Control Letters, Vol. 21, pp. 289-293.

Qu, Z. \& Dorsey, J. (1991) Robust PID control of robots, Int. J. Robot. Automat.,6, 1991, 228-235.

Gorez, R. (1999) Globally stable PID-like control of mechanical systems, Systems and Control Letters, 38,pp. 61-72.

Khalil, H. K. (2002). Nonlinear Systems, 3rd edition. Prentice-Hall, Englewood Cliffs, NJ.

Kelly, R. (1993) "A simple set-point robot controller by using only position measurements", Proc. of the IFAC'93 World Congress, Sydney, Australia, Vol. 6, pp. 173-176.

Kelly, R. (1995) A Tuning procedure for stable PID control of robot manipulators, Robotica, 13(2), pp. 141-148.

Kelly, R.; Santibáñez, V. \& Loria, A. (2005). Control of Robot Manipulators in Joint Space, Springer-Verlag.

Kelly, R. (1998). "Global positioning of robot manipulators via PD control plus a class of nonlinear integral actions ", IEEE Transactions on Automatic Control,Vol. 43, No. 7,pp 934-938.

Kelly, R. \& Ortega, R. (1988). "Adaptive control of robot manipulators: an inputoutput approach" IEEE International Conference on Robotics and Automation, Philadelphia, PA.

Koditschek, D. (1984). "Natural motion for robot arms" Proceedings of the 1984 IEEE Conference on Decision and Control, Las Vegas CA., Dec., pp. 733-735.

Laib, A. (2000). Adaptive output regulation of robot manipulators under actuator constraints. IEEE Transactions on Robotics and Automation, Vol. 16, pp. 29-35.

Landau, I. D. \& Horowitz, R. (1988). "Synthesis of adaptive controllers for robot manipulators using a Passive feedback system approach", IEEE International Conference on Robotics and Automation, Philadelphia, PA.

Loria, A.; Kelly, R.; Ortega \& Santibáñez, V. (1997). On global output feedback regulation of Euler-Lagrange systems with bounded inputs, IEEE Transactions on Automatic Control", Vol. 42, No. 8, pp. 1138-1143.

Meza, J.L.; Santibáñez V. \& Hernandez, V. (2005). Saturated nonlinear PID global regulator for robot manipulators: Passivity based analysis. Proceedings of the 16th IFAC World Congress, Prague, Czech Republic.

Meza, J.L.; Santibáñez V. \& Campa, R.(2007). An estimate of the domain of attraction for the PID regulator of manipulators, Int. J. Robotics Automat. 22, 187-195.

Nijmeijer, H. \& Van der Schaft, A. (1990) "Nonlinear Dynamical Control Systems", SpringerVerlag, NY.

Ortega, R. \& Spong, M. (1989) "Adaptive motion control of rigid robots: a tutorial", Automatica, Vol. 25, No. 6, pp. 877-888.

Ortega, R.; Loria, A. \& Kelly, R. (1995a) A semiglobally stable output feedback PI²D regulator for robot manipulators, IEEE Transactions on Automatic Control, 40 (8), pp. 1432-1436.

Ortega, R.; Loría, A.; Kelly, R. \& Praly, L. (1995b) “On passivity-based output feedback global stabilization of Euler-Lagrange systems" International Journal of Robust and Nonlinear Control, Vol. 5, pp. 313-323.

Orrante, J.; Santibáñez V. \& Campa, R. (2010). On Saturated PID Controllers for Industrial Robots: the PA10 Robot Arm as Case of Study, Advanced Strategies 
for Robot Manipulators, S. Ehsan Shafiei (Ed.), ISBN: 978-953-307-099-5, InTech, Available from: http://www.intechopen.com/articles/show/title/on-saturatedpid-controllers-for-industrial-robots-the-pa10-robot-arm-as-case-of-study.

Ortega, R.; A. Loria, P. J. Nicklasson, P. J. \& Sira-Ramirez, H. (1998). Passivity- based control of Euler-Lagrange systems, in Communications and Control Engineering. Berlin, Germany: Springer.

Ortega, R. \& Garcia-Canseco, E. (2004) Interconnection and damping assignment passivitybased control: A survey, Eur. J Control, vol. 10, pp. 432-450.

Ortega, R.; Vander, A.; Castaños, F. \& Astolfi, A. (2008). Control by Interconnection and Standard Passivity-Based Control of Port-Hamiltonian Systems, IEEE Transaction on Automatic Control, Vol.53,No. 11, pp. 2527- 2542.

Reyes, F \& Kelly, R. (2001). Experimental evaluation of model-based controllers on a directdrive robot arm, Mechatronics,11, 267-282.

Rocco, P. (1996) Stability of PID control for industrial robot arms, IEEE Transactions on Robotics and Automation, 12(4), pp. 606-614.

Santibáñez, V. \& Kelly, R. (1998) “A class of Nonlinear PID Global regulators for robot manipulators", Proc. of 1998 IEEE International Conference on Robotics and Automation, Leuven, Belgium.

Santibáñez, V. \& Kelly, R. (1997) "Strict Lyapunov functions for global regulation of robot manipulators", AUTOMATICA, Vol. 33, No. 4, pp. 675-682.

Santibáñez, V.; Kelly, R.; Zavala-Rio, A. \& Parada, P. (2008). A new saturated nonlinear PID global regulator for robot manipulators, Proceedings of the 17th IFAC World Congress,Seoul, Korea.

Santibáñez, V.; Camarillo, K.; Moreno-Valenzuela, J. \& Campa, R. (2010). A practical PID regulator with bounded torques for robot manipulators. International Journal of Control Automation and Systems, Vol. 8, No. 3, pp. 544-555.

Sepulchre, R.; Jankovic, M. \& Kokotovic, P. (1997). Constructive Nonlinear Control. New York: Springer.

Spong, M.; Hutchinson, M. \& Vidyasagar, M.(2006). Robot Modeling and Control, John Wiley and Sons.

Sun, D.; Hu, S.; Shao, X. \& Liu, C., (2009) Global stability of a saturated nonlinear PID controller for robot manipulators. IEEE Transactions on Control Systems Technology, Vol. 17, No. 4, pp. 892-899.

Takegaki, M. \& Arimoto, S. ( 1981) “A new feedback method for dynamic control of manipulators", ASME Journal of Dynamic Systems, Measurement, and Control, Vol. 103, pp. 119-125.

Tomei, P. (1991). Adaptive PD controller for robot manipulators, IEEE Transactions on Robotics and Automation, 7(4), 565-570.

Van der Schaft, A. (1999). Gain and Passivity Techniques in Nonlinear Control. Berlin, Germany: Springer.

Wen, J. T. \& Bayard, D. S. (1988) “New class of control laws for robotic manipulators. Part 1. Non-adaptive case", International Journal of Control, Vol 47, No. 5, pp. 1361-1385.

Wen, J. T. \& Murphy, S. (1990). PID control for robot manipulators, CIRSSE Document 54, Rensselaer Polytechnic Institute. 


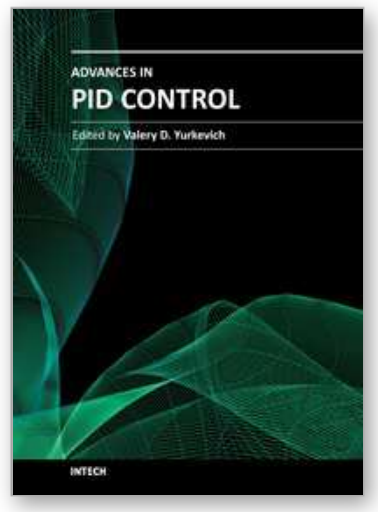

\author{
Advances in PID Control \\ Edited by Dr. Valery D. Yurkevich
}

ISBN 978-953-307-267-8

Hard cover, 274 pages

Publisher InTech

Published online 06, September, 2011

Published in print edition September, 2011

Since the foundation and up to the current state-of-the-art in control engineering, the problems of PID control steadily attract great attention of numerous researchers and remain inexhaustible source of new ideas for process of control system design and industrial applications. PID control effectiveness is usually caused by the nature of dynamical processes, conditioned that the majority of the industrial dynamical processes are well described by simple dynamic model of the first or second order. The efficacy of PID controllers vastly falls in case of complicated dynamics, nonlinearities, and varying parameters of the plant. This gives a pulse to further researches in the field of PID control. Consequently, the problems of advanced PID control system design methodologies, rules of adaptive PID control, self-tuning procedures, and particularly robustness and transient performance for nonlinear systems, still remain as the areas of the lively interests for many scientists and researchers at the present time. The recent research results presented in this book provide new ideas for improved performance of PID control applications.

\title{
How to reference
}

In order to correctly reference this scholarly work, feel free to copy and paste the following:

Jose Luis Meza, Victor Santibáñez, Rogelio Soto, Jose Perez and Joel Perez (2011). Analysis via Passivity Theory of a Class of Nonlinear PID Global Regulators for Robot Manipulators, Advances in PID Control, Dr. Valery D. Yurkevich (Ed.), ISBN: 978-953-307-267-8, InTech, Available from:

http://www.intechopen.com/books/advances-in-pid-control/analysis-via-passivity-theory-of-a-class-ofnonlinear-pid-global-regulators-for-robot-manipulators

\section{INTECH}

open science | open minds

\section{InTech Europe}

University Campus STeP Ri

Slavka Krautzeka 83/A

51000 Rijeka, Croatia

Phone: +385 (51) 770447

Fax: +385 (51) 686166

www.intechopen.com

\section{InTech China}

Unit 405, Office Block, Hotel Equatorial Shanghai

No.65, Yan An Road (West), Shanghai, 200040, China 中国上海市延安西路65号上海国际贵都大饭店办公楼 405 单元

Phone: +86-21-62489820

Fax: +86-21-62489821 
(C) 2011 The Author(s). Licensee IntechOpen. This chapter is distributed under the terms of the Creative Commons Attribution-NonCommercialShareAlike-3.0 License, which permits use, distribution and reproduction for non-commercial purposes, provided the original is properly cited and derivative works building on this content are distributed under the same license. 